\title{
Pasión, oficio y método en la Ciencia Política*
}

\author{
Discurso de apertura del III Congreso Nacional de Ciencia Política \\ de la Asociación Colombiana de Ciencia Política (ACCPOL) \\ 24 de septiembre de 2014
}

\author{
Richard Snyder, Ph.D.**
}

Quiero agradecer y felicitar a mis colegas de la Universidad Icesi y a todos los que hicieron posible el Congreso y mi visita aquí.

Empezaré preguntándo: ¿Por qué eligieron estudiar Ciencia Política?; ¿Por qué les llama la atención la Ciencia Política, en este momento? Piensen en esto. Quizá podamos hablar un poco de eso, quizá la charla que voy a ofrecer los va a convencer de que hicieron una elección correcta, o puede ser al contrario, eso es seguro. Vamos a ver...

Bueno, este es el título de la conferencia Pasión, Oficio y Método: La dimensión humana de la investigación comparada. Realmente es la investigación política, de Ciencias Sociales. Mucho de lo que voy a hablar viene de una serie de entrevistas que publicamos ya hace siete años, en el 2007 con mi colega Gerardo Munck ${ }^{1}$ y lo importante es ¿Quiénes son los entrevistados?

* La transcripción de este discurso de apertura procuró mantener el carácter oral de la misma. Para esta publicación, el autor revisó y enriqueció su contenido. Transcrito por Aura Medina. Revisado y notas a pie de página por Maria A. López.

** Agradecimientos especiales a Richard Snyder, profesor asociado del Instituto Watson de la Universidad de Brown y director del Centro de Estudios de América Latina y el Caribe (CLACS) de la Universidad de Nueva York.

1 Gerardo Munck, Ph.D. es profesor de Relaciones Internacionales de la Escuela de Relaciones Internacionales de la Universidad del Sur de California (USC). 
Es un buen momento para hablar de este libro porque ya salió una edición coreana, va a salir una edición China pero aún no ha salido la edición en español, pero estamos en eso firmemente, parece que va a salir en el año que entra. Entonces, para mí es muy interesante escribir una nueva introducción para la edición en español, puede ser muy interesante tener retroalimentación de ustedes.

En fin, lo importante es: ¿Quiénes son ellos? Esta es la lista de entrevistados, fecha de nacimiento, donde nacieron y es una selección de los politólogos más importantes de los últimos sesenta años. Nombres que espero sean conocidos. Aquí hay algunas fotos, lo bueno es que los entrevistamos antes de que fallecieran.

¿Cuántos han leído a Samuel P. Huntington? Algunos. ¿Barrington Moore, Jr.? Algunos. ¿Robert Dahl? ¡Muchos! Tema de Democracia, muy importante. También tenemos un grupo de extranjeros (bueno, extranjeros para los Estados Unidos) ¿Quién ha leído algo de mi gran amigo Juan J. Linz? También conocido. ¿Guillermo O’Donnell? Muchos. ¿Alfred Stepan? ${ }^{2}$ ? Pocos.

Entonces, es un libro de entrevistas con ellos. Entrevistas muy profundas, sobre su formación personal y por qué ellos decidieron estudiar Ciencia Política y Sociología en algunos casos. Lo que quiero ofrecerles es una destilación de la sabiduría de ellos. Entonces va a ser muy bonito, les ofrezco un coctel, un mojito sabio sobre el valor y el propósito de la Ciencia Social, de la Ciencia Política.

Va a ser muy curioso, la versión coreana ¿alguien lee aquí coreano? Nadie... ¿nadie? Yo tampoco. Bueno, según lo que me han dicho, hicieron un cambio en el título del libro. El título en coreano quiere decir, ¿cómo han hecho para ser los mejores politólogos? Entonces, si escuchan de ellos ustedes también pueden llegar a ser de los mejores politólogos.

Antes de empezar ¿por qué solo hay una mujer en el grupo (Theda Skocpol ${ }^{3}$ )? Es importante hablar un poco sobre eso. Veo aquí casi mitad y mitad, balance de género, de mujeres y de hombres. Igual aquí es por la selección de casos, de gente para entrevistar que usamos, quisimos que fuera gente que ha hecho grandes contribuciones a largo plazo

2 Politólogo comparativista, Ph.D. de la Universidad de California y actual director del Centro de Estudios de la Democracia, Tolerancia y Relgión de la Universidad de Columbia.

3 Socióloga y politóloga norteamericana, profesora Victor S. Thomas de gobierno y sociología de la Universidad de Harvard. 
para la disciplina, por lo tanto, elegimos una fecha de nacimiento, antes de 1950 y resulta que no hubo tantas mujeres entrando al programa de doctorado en Ciencia Política para ser profesor, profesor en Ciencia Política en esa época. Simplemente es una función de los números, del tamaño del grupo. Estoy seguro de que si se hace una edición nueva de algo así, poniendo como fecha de nacimiento 1970-80 sería otro balance de género.

También quiero comentar que unos colegas están haciendo un libro parecido, para América Latina. Un colega peruano Martin Tanaka ${ }^{4}$. Un colega Chileno Manuel Garretón ${ }^{5}$ y Gerardo Munck. Están entrevistando politólogos en toda América Latina. Este es un ejercicio de formación de identidad profesional para cada país. En el caso de ustedes, si quieren buscar los quince mejores politólogos de Colombia ¿Quiénes serían?

Bueno, empecemos a tomar nuestro "coctel sabio" y veamos que se puede aprender de ellos. Entonces, los ingredientes de este coctel son tres. Reflexionando en la calidad de las quince "vacas sagradas" de nuestra Ciencia Política, serían tres los ingredientes: la experiencia, la pasión y el riesgo. Voy a hablar de cada una de ellas detalladamente.

Experiencia de vida, que genera interés en tópicos, en temas de investigación y quizá más importante, que le da al investigador una motivación -hasta personal-o sea que le importe el tema de investigación Que no sea algo abstracto que no le interesa, que no le importa.

Pasión, que a veces sale de la misma experiencia y de los compromisos normativos que tenga el investigador o investigadora. Es importante que el tema pueda tener compromisos normativos, preferencias en ciencia, en la investigación científica, no debe ser neutral y sin vinculación a valores.

Y tercero, los Riesgos son la capacidad de emprender desafíos intelectuales y a veces personales, aunque uno de los entrevistados lo expuso muy bien, cuando dijo "yo no estoy dispuesto a morir por las ciencias sociales" Explicando por qué no quería ir a hacer investigación de campo en Sierra Leona (África) durante la guerra civil.

Hay algo interesante, y no estoy seguro de que sean muy relevantes estos hallazgos de nuestra investigación para ustedes. Hay un consenso entre los 15 entrevistados de que

4 Doctor en Ciencia Política de la FLACSO de México. Actualmente, es profesor asociado de la Pontificia Universidad Católica del Perú e investigador principal en el Instituto de Estudios Peruanos.

5 Licenciado en Sociología en la Universidad Católica de Santiago de Chile y Doctorado de l'Ecole des Hautes Etudes en Sciences Sociales, en París. 
hay una oferta débil o ausente de estas cualidades en los politólogos jóvenes. Pero no sé si eso sea cierto en el caso colombiano.

Bueno, hablamos primero de experiencia. En las entrevistas, los investigadores que entrevistamos hacen una vinculación clara entre sus experiencias de vida y sus motivos de investigación. Hay un ejemplo aquí de Robert Dahl ${ }^{6}$ (él era un joven en los años 3040) quién participaba en la segunda guerra mundial para el ejército estadounidense:

\footnotetext{
"la amenaza de los años treinta era que la democracia podía desaparecer, ser destruida. Ese contexto marcó para nuestra generación, la importancia de la democracia, nos dimos cuenta de que las alternativas a la democracia eran mucho peores"
}

Entonces surge un compromiso de vida con la democracia para Robert Dahl.

El caso de Juan Linz: Se crio en España, nació en Alemania, vivió en Alemania hasta la edad de 8 o 9 (1936) tenía recuerdos de niños del surgimiento de los nazis en Alemania. Se mudó a España justo a tiempo para presenciar la Guerra Civil de España:
“ignorar la Guerra Civil Española, o el régimen de Franco no era concebible para un joven español como yo con interés en la Ciencia Política y en las cien- cias sociales. Dada tu historia de vida, tienes un interés personal y un compro- miso que motiva la selección de muchos problemas de investigación" (Linz)

Entonces, la biografía puede incidir en la investigación en la selección de temas de investigación.

Hay otros ejemplos, por ejemplo Alfred Stepan. Cuando terminó los estudios de pregrado en 1957 tomaba un gran viaje a través de todo el mundo por nueve meses. Claro, su familia tenía los recursos para apoyarle en esto y lo hizo. Fue a la India, fue a Indonesia, Pakistán, y en ese momento estuvo expuesto a culturas y religiones diferentes. Después de eso entró en el ejército de Estados Unidos, fue marinero por tres años, después reportero. Si ustedes conocen a Stepan, saben que es muy reconocido por su trabajo sobre el

6 Fue uno de los politólogos contemporáneos más representativos de la disciplina. Autor del célebre libro $L a$ poliarquía: participación y oposición (1997) 
ejército, su primer libro que salió de la tesis de doctorado bajo la supervisión de J. Linz sobre el golpe militar en Brasil en 1964. Su equipo de trabajo y asesores le dijeron que no podía hacer eso, que nunca iba a tener acceso a los generales y coroneles del ejército brasilero, pero tomo un riesgo (otra cualidad) y dijo "bueno, voy a hacerlo aunque me digan que no puedo hacerlo, mis asesores, y voy a enseñarles que si es posible" y lo hizo, encontró la forma y su preparación como un ex militar estadounidense le permitió poder hablar con los militares brasileños durante este régimen militar de los sesentas.

Robert H. Bates ${ }^{7}$, fue a África cuando era alumno de secundaria y eso le cambio la vida. Dijo que quería radicarse en África y así lo hizo, estudia la región del África. El escribió sobre Colombia, estuvo en Colombia en el sector cafetero, Manizales. Es interesante que no lo conozcan ustedes. Entonces desde la idea de que hay una vinculación entre la biografía y los temas de investigación surge una hipótesis un poco provocadora e interesante: que la calidad de la investigación puede depender de la calidad de las experiencias de cada uno.

Como ya he dicho, la experiencia de vida puede dar a la investigación sentido y propósito, también puede ser una fuente de nuevas ideas. Porque si conoces algo a fondo encuentras teoría, pero la teoría no corresponde con la realidad que conoces; allí hay una fricción muy fértil para la investigación. Les doy unos ejemplos: Linz, conocía muy bien el caso de España bajo la dictadura del General Franco y sabía que la naturaleza de este régimen no correspondía a la categoría de totalitarismo. Lo sabía basado en la experiencia propia en España. Otro caso, es el del holandés Arend Lijphart. Holanda es un país multicultural, pero también es una democracia estable, en los años sesenta cuando él estaba estudiando el doctorado en Yale, la teoría dijo que en países donde los clivajes son así, no es posible una democracia estable, pero el caso holandés desmentía esa teoría.

El sociólogo político Seymour Martin Lipset ${ }^{8}$, decía que un mecanismo que conecta intereses al sistema político, es el partido político. Vincula valores e intereses sociales al sistema político. Sin embargo, Philippe C. Schmitter ${ }^{9}$ sabia que en Suiza no era así, el partido político no tenia ninguna incidencia. En suiza èstos no representaron intereses,

7 Actualmente es Profesor Eaton del Departamento de Gobierno, y miembro del Departamento de Estudios Africanos y Africano-americanos de la Universidad de Harvard.

8 Es autor de El hombre político (1960), Revolución y contrarrevolución (1968), Consenso y conflicto (1985).

9 Profesor Emérito del Departmento de Ciencias sociales y política del Instituto Universitario Europeo. 
sino asociaciones de interés. Entonces la experiencia es una forma de abordar la teoría, cuando no coinciden. ¿Tiene usted experiencia?

\footnotetext{
"Los jóvenes estudiando Ciencia Politica, por haber crecido en un mundo pacifico, prospero, en tiempos libres de conflicto, quizá un mundo post conflicto, que son inteligentes, educados pero no tienen experiencia ni interés. Estos jóvenes absorben la educación y obtienen capacidades fácilmente, pero cuando el momento llega y tienen que hacer preguntas, ellos no tienen nada que preguntar", (Adam Przeworski)
}

¡No me gustaría tenerlo a él como mi papá!

Entonces son inquietudes de los entrevistados, sobre el capital humano que está entrando en el momento en Estados Unidos, por lo menos. Entonces ¿qué se puede hacer frente a la falta de experiencia? Una cosa es, no ir directamente a estudiar posgrados, si es que están pensando eso, justo después de terminar el pregrado. ¡Toma tiempo para trabajar! Obviamente si se puede hacer algo diferente, salir del sendero académico. Otra forma de ganar experiencia es hacer investigación de campo "El trabajo de campo es la cura para la generación de conclusiones sin sustento, cuando se hace trabajo de campo los problemas de investigación se obtienen de la realidad" (Bates).

Es bueno sentirse outsider, vivir en otra cultura, y no tiene que ser en otro país. Dentro de la misma ciudad, o lugar donde uno habita hay una variación impresionante. Estamos en esta parte de Cali, yo desconozco la ciudad, pero tengo entendido que no estamos en la zona oriental de la ciudad que se llama Aguablanca ¿cómo está esa zona de la ciudad de Cali? ¿Alguien lo conoce? ¿Cómo es? Sinceramente, yo desconozco. Tengo entendido que es muy distinto del hotel y de la ciudad. Espero que el punto quede claro, que no hay que viajar para hacer comparaciones, se puede dentro de la misma ciudad, la diferencia puede ser de cultura, socioeconómica, etc.

Llegamos a la segunda característica de los investigadores destacados que es la pasión. Aquí tienen un dicho que es de Sherlock Holmes "Soy un cerebro, el resto de mi es mero apéndice” hay otra perspectiva que nos ofrece Max Weber "la inspiración, pasión, juega en la ciencia un papel tan importante, como el que juega en el arte". Las entrevistas acuerdan mucho más con Weber que con Sherlock Holmes, se nota que son muy apasionados. 
Otro ejemplo de pasión viene de Guillermo O’Donnell, dice que le gusta enfocarse en los problemas que le molestan en la mañana cuando está afeitándose. Tuvo una barba durante unos años, entonces no estoy seguro de que hizo durante aquellos años, pero bueno... Quizá estaba más tranquilo. El tenía una obsesión, obviamente, con su país Argentina. Robert Dahl nos ofrece lo siguiente: "Para los mejores alumnos, estudiar política no solo es algo que involucra su cerebro sino también su sistema somático, sentimientos”. No siempre, no el cien por ciento del tiempo. ¿Qué es lo que motiva su pasión por la investigación? Bueno, a veces como dije se divierten, es divertido, ¡les gusta! Se ponen como niños de alguna forma.

Otra fuente de pasión, puede ser la vinculación a los valores del investigador. Aquí dice Lijphart que el empieza normativamente, con una variable muy importante, algo que puede ser descrito como bueno o malo, como la violencia o la paz. Luego sigue investigando que produce estos diferentes resultados y finalmente, concluye presentando prescripciones. $\mathrm{O}$ sea medidas que pueden producir resultados deseados, Lijphart no vee una tensión entre preocupaciones normativas y la intención de hacer ciencia.

Otra vez encontramos en las entrevistas, una preocupación de que estudiantes de posgrado no tienen pasión, o tienen menos pasión. Skocpol nos dice:

"hablo con muchos estudiantes de postgrado que dicen sentirse muy limitados. Parece que ellos eligen preguntas motivados por un sentido del deber, trabajando en un tema en particular, porque es lo que se espera que hagan para alcanzar la siguiente etapa profesional, no estoy suficientemente segura de que esta gente esté siguiendo sus instintos y creyendo que su curiosidad los llevará a una pregunta importante".

James C. Scott dice que "demasiados profesores y alumnos hoy, piensan que la investigación es una carrera, un trabajo de nueve a cinco” ¿por qué eligen estudiar ciencia política? ¿Por cuál motivo? Y hay muchos motivos naturalmente aceptables, pero desconozco este contexto entonces. Eso es pregunta para ustedes.

Finalmente, porque creo que ya es bueno que termine de hablar, llegamos a la última cualidad que se destaca a los entrevistados, tomar riesgos. Riesgos en varios aspectos, en términos de cómo definen sus relaciones con los maestros, también en cómo se define su relación con el mainstream y también tomar riesgos en términos de las preguntas que 
deciden investigar. Por ejemplo Skocpol, su primer artículo, ella lo publicó cuando era alumna de posgrado, y era una crítica a uno de sus asesores más importantes. Como les dije ya, Stepan renuncio a la asesoría cuando le dijeron que no era posible estudiar al ejército brasilero durante el régimen militar durante los años sesenta.

Otro ejemplo es Scott. Decidió después de su primer libro, basado en su tesis doctoral sobre los elites politicos en Malasia, y que no considero un trabajo sólido, le gustó sus asesores por la metodología pero para el no era muy bueno porque no tenía conocimiento profundo del contexto, entonces el emplea toda la energía en entender profundamente el contexto agrícola de la cultura campesina de Asia y resulta que va allá con permanencia, se va a vivir dos años a un pueblo en Malasia para ser un campesino y hacer lo que resulta que quizá es su mejor libro, Weapons of the Weak. Uno de sus colegas en ese momento le dijo lo siguiente: que era muy tonto porque invertir en profundizar su conocimiento de Malasia era un desgaste de tiempo, que no era hacia donde la Ciencia Política estaba yendo, que era el fin de su carrera, según este colega, y resulto todo lo contrario, salió de esto su libro más conocido.

Demasiado profesionalismo, puede ser limitado, rígido y limitar la visión. No sé si eso ocurra en el contexto colombiano, pero es una imagen de lo que puede ocurrir en un futuro. Demasiado profesionalizados. Y aquí viene O'Donnell con su preocupación hacia la sofisticación metodológica "la Ciencia Política ha perdido la ambición de escribir libros grandiosos que den cuenta de grandes problemas".

Skocpol dice "diversificación, no pegarse a estudiar solo con una sola persona o escuela, sino varias. Así se da la posibilidad de crear una combinación original”

Bueno, concluyo con una lista de recomendaciones que vienen de los entrevistados. Es un resumen de lo que he hablado:

- Salir de la senda académica y tener experiencias en el mundo real.

- Hacer trabajo de campo.

- Estudiar con profesores apasionados en la investigación y que no vean el trabajo académico como un trabajo de 9:00 a 5:00.

- Construir oportunidades fuertes e interactivas con otros estudiantes y profesores que se salgan de los límites del salón de clases y del entrenamiento formal.

- No temer que los compromisos normativos y los valores determinen la selección de 
problemas de investigación. No temer explorar las implicaciones normativas del trabajo.

- Tomar riesgos mesurados.

- Intenta disfrutar y divertirte con la investigación, y ser politólogo. No siempre es felicidad, pero hay que intentarlo.

Bueno, termino con eso. Gracias por su atención. 
